\title{
Absceso y flegmón periamigdalino ¿Lo estamos haciendo bien?
}

\section{Abscess and cellulitis peritonsillar ¿Are we doing it right?}

\author{
César Gómez V1․ Edda Pruzzo Ch${ }^{1}$, Eduardo Reyes $\mathrm{S}^{2}$, María de los Angeles Contreras $\mathrm{R}^{2}$, \\ José Miguel Contreras $\mathrm{R}^{1}$, Viviana Vergara $\mathrm{T}^{1}$.
}

\begin{abstract}
RESUMEN
日 flegmón (celulitis) y el absceso periamigdalino son infecciones difusas o una colección ubicada entre la cápsula fibrosa de la amígdala palatina, las fibras horizontales del músculo constrictor superior de la faringe y las verticales del músculo palatofaríngeo. Constituye la infección de tejidos y espacios profundos del cuello más frecuente.

Material y método: Revisión retrospectiva de las fichas de pacientes adultos y niños hospitalizados con diagnóstico de absceso o flegmón periamigdalino en el Servicio de Corrinolaringología del Hospital San Juan de Dios entre los años 1995 y 2001.

Resultados y discusión: Se analizaron 124 pacientes. Se observó un acierto del diagnóstico clínico en $85,5 \%$ de los casos. 1 100\% de los pacientes presentó evolución clínica favorable según la modalidad terapéutica indicada La mayoría de los pacientes con absceso periamigdalino fue tratado con drenaje y antimicrobiano, y los con flegmón periamigdalino con antimicrobiano. $B$ antibiótico utilizado con mayor frecuencia fue penicilina No existió diferencia significativa al usarlo en esquema asociado. La penicilina sódica sigue siendo un antimicrobiano de primera elección para este cuadro y no requeriría de asociaciones; dosis de 3 millones de Ul cada 6 horas endovenosa pueden ser recomendadas. $\theta$ tratamiento en el Servicio de Ctorrinolaringología del Hospital San Juan de Dios se ciñe a las reglas internacionales.
\end{abstract}

Palabras Clave: Absceso periamigdalino, flegmón periamigdalino, diagnóstico, cultivo, tratamiento.

\section{SUMMARY}

Cellulitis and abscess peritonsillar are a diffuse infection or a collection located between the fibrous capsule of the palatine tonsil, the horizontal fibers of the superior constrictor muscle of the pharynx and the vertical ones of the palatopharyngeal muscle. It is most frequently an infection of deep tissue and spaces of the neck.

Material and method: Retrospective review of the index cards of adult patients and children hospitalized with diagnosis of abscess or peritonsillar phlegmon cellulitis at the Aolaryngology Service of Hospital San Juan de Dios between years 1995 and 2001.

\footnotetext{
${ }^{1}$ Médico del Servicio de Otorrinolaringología, Hospital San Juan de Dios.

${ }^{2}$ Alumno de $7^{\circ}$ año de Medicina, Universidad de Chile
} 
Results and discussion: 124 patients were analyzed. In $85 \%$ of the cases good judgment of the clinical diagnosis was noticed. $100 \%$ of the patients showed favorable clinical evolution according to the therapeutic mode indicated. The majority of the patients with peritonsillar abscess were treated with drainage and antimicrobial, and those with peritonsillar phlegmon with antimicrobia. The antibiotic most frequently used was penicillin. No significant difference was observed when using it in associated scheme. Sodium penicillin continues to be a first selection antimicrobial for this picture and would nor require associations; endovenous doses of 3 million Ul every 6 hours may be recommended. Treatment at the Colaryngology Service of Hospital San Juan de Dios follows the International rules.

Key words: Peritonsillar Abscess, peritonsillar cellulitis, diagnosis, cultive, treatment.

\section{INTRODUCCIÓN}

日 absceso periamigdalino (AP) y el flegmón (celulitis) periamigdalino $(\mp)$ constituyen la infección de tejidos y espacios profundos del cuello más frecuente en la población adulta, con aproximadamente 30 casos cada 100 mil habitantes ${ }^{1}$. Fue descrito por primera vez durante el imperio romano, aunque no fue hasta el siglo XIV, cuando el cirujano francés, Guy de Chaliac, describió su drenaje con la técnica que no ha sufrido cambios hasta la actualidad?

Es un cuadro capaz de originar morbilidad grave como fascitis necrotizante y mediastinitis, así como mortalidad elevada ${ }^{3-6}$. Por ello, exige un diagnóstico temprano y un tratamiento efectivo que impidan la extensión a otros espacios del cuello.

Se entiende por AP y 円 una infección, difusa (flegmón o celulitis) o colección (absceso), ubicada entre la cápsula fibrosa de la amígdala palatina, las fibras horizontales del músculo constrictor superior de la faringe y las verticales del músculo palatofaríngeo ${ }^{1}$. Algunos autores no lo consideran infección de espacio profundo de cuello ${ }^{5}$.

A pesar de ser una patología frecuente, no existe un tratamiento único para abordarlo. Diversos procedimientos utilizados incluyen el uso de antimicrobiano (ATM) endovenoso más punciones aspirativas repetidas, ATM más drenaje quirúrgico, ATM más punción y drenaje, amigdalectomía durante el proceso infeccioso mismo ("en caliente"), amigdalectomía diferida, etc., existiendo gran cantidad de publicaciones extranjeras que avalan las ventajas de uno y otros ${ }^{1,2,7-11}$.
Con el fin de averiguar cuál es la realidad local respecto al diagnóstico, tratamiento y frecuencia de complicaciones, y compararla con publicaciones internacionales, se realizó un análisis retrospectivo de los pacientes con diagnóstico de AP y 円 ingresados al Servicio de Otorrinolaringología del Hospital San Juan de Dios entre los años 1995 y 2001.

\section{MATERIAL Y MÉTODOS}

Se revisaron las fichas de los pacientes adultos y pediátricos hospitalizados con diagnóstico de AP o 円 en el Servicio de Otorrinolaringología del Hospital San Juan de Dios entre el $1^{\circ}$ de enero de 1995 y el 31 de diciembre de 2001.

Los pacientes incluidos refirieron un cuadro clínico de curso agudo, sintomatología inflamatoria orofaríngea y presencia de aumento de volumen periamigdalino (abombamiento).

Se definió AP en los pacientes que se describió a su ingreso abombamiento severo de la región orofaríngea más la existencia de material purulento espontáneo o al efectuar el drenaje, y $\mathrm{T}$ como el abombamiento moderado de la región orofaríngea o a la ausencia de obtención de material purulento espontáneo o al drenaje. Se descartaron los pacientes con otros diagnósticos orofaríngeos y/o infección de otros espacios profundos de cuello.

En el período estudiado se registraron 176 ingresos con el diagnóstico de AP o P. De los 176 ingresos, 52 fueron descartados por datos incompletos, resultando un grupo final de 124 pacientes. 
Se confeccionó una ficha tipo para el registro de parámetros epidemiológicos, clínicos y terapéuticos.

Para evaluar la evolución clínica, se tomaron en cuenta 4 parámetros: días de hospitalización, trismus, abombamiento palatino y fiebre. Se consideró que un paciente respondía satisfactoriamente a un esquema o dosis de ATM si a las 48 horas de hospitalización, estos parámetros clínicos desaparecían o disminuían notoriamente.

La amigdalectomía, siempre diferida, fue indicada en aquellos pacientes con más de un episodio de AP o $\mathrm{P}$, o los que presentaron un primer episodio de AP $\circ \mathrm{P}$ más historia de faringoamigdalitis recurrente.

日 análisis de datos se efectuó con los progra mas estadísticos de Epi-Info 6.04 y STATA.

\section{RESULTADOS}

Los 124 pacientes estudiados correspondieron al $2,1 \%$ del total de los pacientes hospitalizados en el período 1995-2001. 日 61,3\% correspondió al gé nero femenino, y el 38,7\% al masculino. $日 20,2 \%$ de los pacientes era menor de 15 años, y el 79,8\% mayor de esa edad.

日 proceso infeccioso no presentó diferencia en lateralización (lado derecho 60 pacientes, izquierdo 63, un paciente sin dato).

A ingreso, 84 pacientes fueron diagnosticados clínicamente como AP, pero en sólo 78 casos se confirmó el diagnóstico al ser positivo el drenaje (92,9\% de acierto). Quarenta pacientes fueron ingresados con el diagnóstico clínico de P, y en 28 casos se confirmó el diagnóstico ( $70 \%$ de acierto). En los 12 restantes se confirmó la presencia de absceso. $\mathrm{日}$ porcentaje de acierto conjunto con el diagnóstico al ingreso fue de $85,5 \%$.

日 promedio de días entre el inicio de síntomas y la fecha de hospitalización fue de 4 días para los AP (1-14 días), y 3 días para los $円$ (1-8 días).

En $68,5 \%$ de los pacientes, éste fue su primer episodio de PP o AP y 19,4\% refirió episodios anteriores de PP o AP. En 12,1\% la información no fue consignada en la ficha.
Respecto al antecedente de amigdalitis recurrente, 22 de los pacientes $(24,4 \%)$ con AP lo refirieron y $14(41,2 \%)$ de los con $\mathrm{P}$, sumando un total de $36(29 \%)$ de los 124 pacientes.

Solamente un paciente padecía de diabetes mellitus tipo 2 y ninguno de ellos enfermedad inmunológica conocida (Tabla 1).

日 $77,8 \%$ de los pacientes con AP y el $80,8 \%$ de los con $\mathrm{P}$ recibieron tratamiento antibiótico previo a la hospitalización que pudo considerarse adecuado para el tratamiento de faringoamigdalitis estreptocócica de acuerdo al ATM seleccionado, dosis y duración.

日 tratamiento intrahospitalario de los pacientes fue el siguiente: todos los pacientes con AP se trataron con antibioticoterapiay algún procedimiento. Punción más incisión y drenaje en el $15,6 \%$ de los casos, sólo incisión y drenaje en el $84,4 \%$. En los $\mathrm{P}$, en $41,2 \%$ de los casos el tratamiento consistió sólo en antibioticoterapia, y en el $58,8 \%$ de los casos el tratamiento incluyó algún procedimiento (punción en el 11,8\%, punción más incisión y drenaje en el 2,9\%, solo drenaje en el $44,1 \%$ de los casos). De un total de 40 pacientes que ingresaron con diagnóstico de $円 \mathrm{Pl}$ ingreso, en 12 se confirmó la existencia de un AP luego del procedimiento (Figuras 1 y 2 ).

Respecto al tiempo de hospitalización, en los pacientes con $\mathrm{PP}$ (34 pacientes), el promedio de estada fue de 5 días (intervalo de 3-12 días). En el caso de los pacientes con AP (90 pacientes), el período de permanencia fue similar, con un promedio de 5,5 días (intervalo de 1-10 días).

A analizar el ATM utilizado, este fue penicilina endovenosa en el $86 \%$, penicilina endovenosa más alguna asociación en el $11 \%$ y clindamicina

Tabla 1. Antecedentes mórbidos en 124 pacientes con AP o FP

\begin{tabular}{|lcr|}
\hline & Número & $(\%)$ \\
& de pacientes & \\
\hline Episodio anterior AP o $\mathrm{P}$ & 24 & $(19,4)$ \\
Amigdalitis recurrente & 36 & $(29)$ \\
Diabetes mellitus & 1 & $(0,8)$ \\
Enfermedad inmunológica & 0 & $(0)$ \\
\hline
\end{tabular}




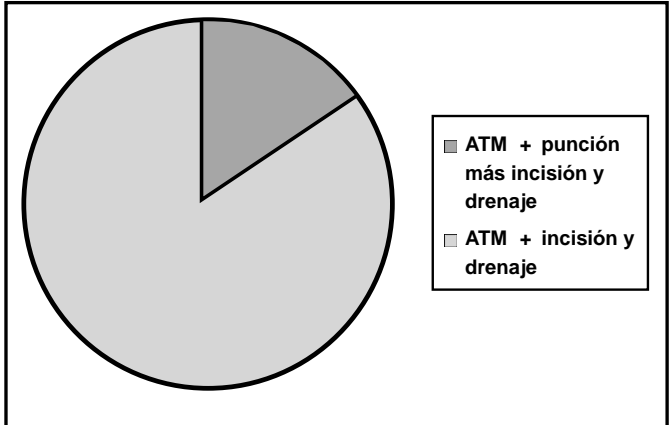

Figura 1. Tratamiento intrahospitalario en pacientes con AP. ATM =antibioticoterapia.

endovenosa en el 3\% de los pacientes. Los datos respecto a las dosis utilizada se pudieron obtener desde la ficha clínica en 115 de 124 pacientes.

La elección del esquema antibiótico entre penicilina sódica en dosis de 3 millones, 4 millones o 5 millones cada 6 horas se realizó de forma indistinta y dependió exclusivamente del médico que realizó el ingreso del paciente. Con los tres esquemas se

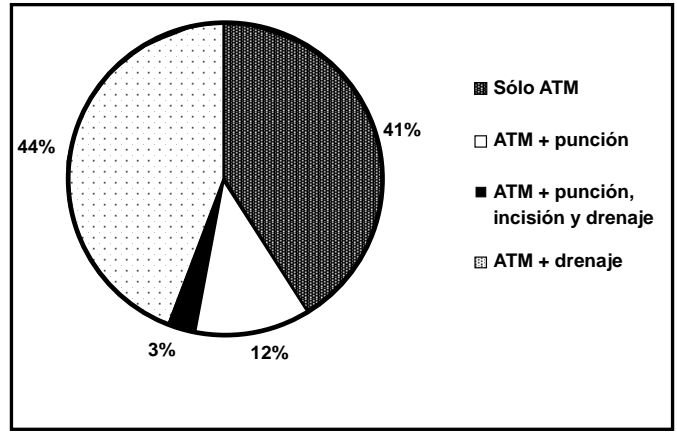

Figura 2. Tratamiento intrahospitalario en pacientes con $\mathrm{P}$. ATM =antibioticoterapia.

observó adecuada regresión de la sintomatología, incluso una mejor tendencia en ellos con dosis de 4 millones. (Tabla 2).

Todos los pacientes presentaron evolución clínica favorable de acuerdo a los parámetros definidos de: días de hospitalización, trismus, abombamiento palatino y fiebre. Para comparar la evolución de los pacientes según la terapia empleada se analizó la

Tabla 2. Flegmón y absceso periamigdalino. Mejoría de los parámetros clínicos a las 48 horas de evolución según antimicrobiano utilizado en 115 pacientes

\begin{tabular}{|c|c|c|c|c|c|c|c|c|c|}
\hline & \multicolumn{3}{|c|}{ Ausencia de Trismus N (\%) } & \multicolumn{3}{|c|}{$\begin{array}{l}\text { Ausencia de abombamiento } \\
\text { de paladar } \mathrm{N}(\%)\end{array}$} & \multicolumn{3}{|c|}{$\begin{array}{l}\text { Ausencia de fiebre } \\
\qquad \mathrm{N}(\%)\end{array}$} \\
\hline & $A$ & $\mathrm{~F}$ & No evaluable ${ }^{*}$ & A & $\mathrm{F}$ & No evaluable* & A & $\mathrm{F}$ & No evaluable* \\
\hline $\begin{array}{l}\text { PNC SOD } \\
3 \text { millones } \\
\text { ( } 36 \text { pacientes) }\end{array}$ & $\begin{array}{c}12 \\
(33,3)\end{array}$ & $\begin{array}{c}9 \\
(25)\end{array}$ & $\begin{array}{c}3 \\
(8,3)\end{array}$ & $\begin{array}{c}16 \\
(44,4)\end{array}$ & $\begin{array}{c}12 \\
(33,3)\end{array}$ & $\begin{array}{c}3 \\
(8,3)\end{array}$ & $\begin{array}{c}15 \\
(41,6)\end{array}$ & $\begin{array}{c}6 \\
(16,6)\end{array}$ & $\begin{array}{c}10 \\
(27,7)\end{array}$ \\
\hline $\begin{array}{l}\text { PNC SOD } \\
4 \text { millones } \\
\text { (12 pacientes) }\end{array}$ & $\begin{array}{c}5 \\
(41,6)\end{array}$ & $\begin{array}{c}4 \\
(33,3)\end{array}$ & $\begin{array}{c}0 \\
(0)\end{array}$ & $\begin{array}{c}5 \\
(41,6)\end{array}$ & $\begin{array}{c}4 \\
(33,3)\end{array}$ & $\begin{array}{c}0 \\
(0)\end{array}$ & $\begin{array}{c}5 \\
(41,6)\end{array}$ & $\begin{array}{c}4 \\
(33,3)\end{array}$ & $\begin{array}{c}2 \\
(16,6)\end{array}$ \\
\hline $\begin{array}{l}\text { PNC SOD } \\
5 \text { millones } \\
\text { ( } 67 \text { pacientes) }\end{array}$ & $\begin{array}{c}28 \\
(41,7)\end{array}$ & $\begin{array}{c}7 \\
(10,4)\end{array}$ & $\begin{array}{c}5 \\
(7,4)\end{array}$ & $\begin{array}{c}24 \\
(35,8)\end{array}$ & $\begin{array}{c}7 \\
(10,4)\end{array}$ & $\begin{array}{c}4 \\
(5,9)\end{array}$ & $\begin{array}{c}26 \\
(38,8)\end{array}$ & $\begin{array}{c}7 \\
(10,4)\end{array}$ & $\begin{array}{c}29 \\
(43,2)\end{array}$ \\
\hline
\end{tabular}

$\mathrm{A}=$ absceso periamigdalino. $\mathrm{F}$ = flegmón periamigdalino. * =Parámetro imposible de evaluar desde la ficha clínica. PNC SOD= penicilina sódica. 
presencia de trismus, abombamiento y fiebre a los dos y más días de evolución (Tabla 2).

Se enviaron muestras para cultivo microbiológico en sólo 16,1\% de los casos (20 pacientes) que fueron sometidos a algún procedimiento de drenaje. Según registro en la ficha médica se obtuvo informe del examen en sólo 5 pacientes. En ningún caso el médico tratante especificó la microbiología encontrada

Ninguno de los pacientes de esta muestra fue estudiado con imágenes.

Sólo un paciente presentó eritema nodoso como complicación atribuible al AP o 円.

Respecto al control posterior al alta, 32 pacientes $(25,8 \%)$ tuvieron control consignado en la ficha

Del total de pacientes analizados $37,9 \%$ tuvo indicación quirúrgica, 49,2\% no correspondió indicación operatoria, en tanto que en el $12,9 \%$ no fue posible encontrar la información pertinente. Del total de pacientes con indicación quirúrgica, solamente 19 casos (40,4\%) había sido intervenido al momento de finalizada esta revisión.

\section{DISCUSIÓN}

Los datos epidemiológicos concuerdan con aquellos publicados ${ }^{2}$. La muestra analizada constituyó un número adecuado, superior a algunos reportes $2,11,12$. Sin embargo, el registro de datos incompletos en las fichas clínicas no permitió un análisis de todos los parámetros planificados.

En este estudio, el antecedente de amigdalitis recurrente no fue frecuente en los pacientes con AP $(24,4 \%)$. 日 $50 \%$ de los pacientes con $円$ tenía este antecedente.

日 uso de terapia ATM adecuada previo a la hospitalización no impidió la evolución hacia un AP o P. Esto concuerda con reportes de otros autores como Gidley y cols, en cuya publicación el 50\% de los pacientes con infección de espacios profundos del cuello había recibido terapia antimicrobiana previa a la hospitalización ${ }^{5}$. Sin embargo, un estudio randomizado realizado en niños con faringoamigdalitis que compara el tratamiento con penicilina oral por 7 días versus 3 días y placebo, demostró que el porcentaje de infección del espa- cio peritonsilar es mayor en el grupo placebo ${ }^{13}$.

Se observó acierto global en el diagnóstico clínico en $85,5 \%$ de los casos. Por otro lado, de los 40 pacientes con diagnóstico clínico de $\mathrm{P}, 12$ de ellos correspondieron verdaderamente a un absceso. Esto indica que ante el diagnóstico clínico de $\mathrm{P}$ debe efectuarse un procedimiento complementario para intentar confirmarlo, como son punción, incisión y drenaje, o tomografía computada de cuello ${ }^{14}$.

En los 124 pacientes analizados en este estudio el tratamiento efectuado en base a un diagnóstico clínico, sin complementación con imágenes, tuvo resultados favorables. Cabe destacar que los pacientes con sospecha de infección con compromiso de otros espacios profundos de cuello no fueron incluidos en este estudio.

Los antibióticos utilizados con mayor frecuencia fueron: penicilina endovenosa u oral, clindamicina, amoxicilina, cefalosporinas y macrólidos, ampicilina-sulbactam. No existió diferencia significativa al utilizar cualquiera de éstos ${ }^{15,16}$. Según los resultados obtenidos, la penicilina sódica sigue siendo un antimicrobiano de primera elección para este cuadro y no requiere de asociaciones ${ }^{12}$. Una dosis de 3 millones de Ul cada 6 horas endovenosa es suficiente para tener una buena evolución.

La utilidad clínica del cultivo en este estudio fue nula debido a la baja frecuencia de solicitud y el escaso porcentaje con resultado disponible para determinar modificación en la elección antimicrobiana La elección o modificación de ATM en los pacientes se basó en aspectos clínicos y no en resultados microbiológicos. En pacientes inmunocomprometidos el aislamiento microbiológico constituye un elemento de importancia. $\mathrm{日}$ cultivo debe ser solicitado solamente en pacientes inmunocomprometidos o con historia de absceso recurrente $^{2,15}$.

La indicación de procedimientos de punción más drenaje o de incisión más drenaje está ampliamente avalada por la literatura.

En resumen, podemos concluir que el tratamiento en el Servicio de Otorrinolaringología del Hospital San Juan de Dios se ciñe a las reglas internacionales, con muy buenos resultados en el grupo estudiado logrando 100\% de curación y sólo 
un paciente con complicación atribuible al AP.

Aspectos interesantes de estudios futuros en nuestro medio debieran incluir: identificación de parámetros de severidad clínico y pronóstico, comparación de tratamientos ambulatorio versus hospitalizado, efectividad de punción aspirativa versus incisión y drenaje, efectividad del tratamiento antimicrobiano oral posterior al endovenoso, evolución clínica a largo plazo del paciente tratado por AF periamigdalino, comparación de la amigdalectomía inmediata versus diferida en pacientes con indicación de amigdalectomía en AP o P.

\section{BIBLIOGRAFÍA}

1. JOHNSON R, StEWART M, WRIGHT C. An evidence-based review of the treatment of peritonsiIlar abscess. Otolaryngol Head and Neck Surg 2003; 128 (3): 332-43.

2. Herzon F. Peritonsillar abscess: Incidence, current management practices, and a proposal for treatment guidelines. Laryngoscope 1995; 105 (Suppl 74): 1-17.

3. Dastenies C, RaHal M, ArRegul R. Fascitis necrotizante de cuello. Caso clínico. Rev Otorrinolaringol Or Cabeza y Ouello 2004; 64: 247-51.

4. RidDer GJ, TECHNAU-IhlingK, SANDer A, BOEDE KER CC. Spectrum and management of deep neck space infections: an 8-year experience of 234 cases. Otolaryngol Head Neck Surg 2005; 133 (5): 709-14.

5. GDLEY PW, GHORAYmBY, STI porary management of deep neck space infections. Colaryngol Head Neck Surg 1997; 116(1): 16-22.

6. MarRa S, Hotaling AJ. Deep neck infections. Am J Otolaryngol 1996; 17 (5): 287-98.
7. Sohrafr S, Mognn J, Derkay C. Peritonsillar abscess in children: a 10-year review of diagnosis and management. Int $J$ Pediatr Otorhinolaryngol 2001; 57: 213-8.

8. RAUT V, YUnG M. Peritonsillar abscess: The rationale for interval tonsillectomy. ENT J2000; 79 (3): 206-9.

9. SUZUKI M, UeYAMA T, MOG G Immediate tonsillectomy for peritonsillar abscess. Auris Nasus Larynx 1999; 26: 299-304.

10. PASSY V. Pathogenesis of peritonsillar abscess. Laryngoscope 1994; 104:185-190.

11. MaHARAJ D, RajaH V, HeMSLEY S. Management of peritonsillar abscess. J Laryngol Otol 1991; 105(9): 743-5.

12. KI由t DA, BhatTacharyya N, SiEge NS, SalmaN SD. Selection of antibiotics after incision and drainage of peritonsillar abscesses. Otolaryngol Head Neck Surg 1999; 120 (1): 57-61.

13. ZWART S, ROVERS MM, DEMEKER RA, HOES AW. Penicillin for acute sore throat in children: randomized, double blind trial. BMJ 2003; 327 (7427): 1324.

14. SOOT PM, LOFIUS WK, lO KEW J, AHWA A, YUE V, VAN HASSET CA. Diagnosis of peritonsillar infections: a prospective study of ultrasound, computerized tomography and clinical diagnosis. $J$ Laryngol Col 1999; 113(3): 229-32.

15. CherUKURI S, Benninger MS. Use of bacteriologic studies in the outpatient management of peritonsillar abscess. Laryngoscope 2002; 112(1): 18-20.

16. YILMAZ T, UNAL OF, FGEN G, AKYOL MU, AYAS K. A comparison of procaine penicillin with sulbactam-ampicillin in the treatment of peritonsiIlar abscesses. Eur Arch Otorhinolaryngol 1998; 255(3): 163-5. 